\title{
Temporal shaping of entangled photons
}

\author{
Avi Pe'er, Barak Dayan, Asher A. Friesem, and Yaron Silberberg \\ Department of Physics of Complex Systems, Weizmann Institute of Science, Rehovot 76100, Israel
}

\begin{abstract}
We experimentally demonstrate shaping of the two-photon wavefunction of entangled photonpairs, utilizing coherent pulse-shaping techniques. By performing spectral-phase manipulations we tailor the two-photon wavefunction exactly like a coherent ultrashort pulse. To observe the shaping we perform sum-frequency generation (SFG) with an ultrahigh flux of entangled photons. At the appropriate conditions, SFG performs as a coincidence detector with an ultrashort response time $(\sim 100 \mathrm{fs})$, enabling a direct observation of the two-photon wavefunction. This property also enables us to demonstrate background-free, high-visibility two-photon interference oscillations.
\end{abstract}

PACS numbers: 42.50.Dv, 42.65.Ky, 42.50.St, 03.67.-a

Entangled photons generated by parametric downconversion can exhibit nonclassical correlations between various physical properties such as polarization, momentum and energy, and have been the primary tool in quantum communication science [1, 2, 3, 4, 5]. While the polarization state of polarization-entangled photons has been readily manipulated in various quantum-optics experiments (see, for example [3, 4, 5]), no control over their temporal properties has been demonstrated to date, except the prolongation of their correlation-time by spectral filtering with narrowband filters $[6,7,8]$.

Here we demonstrate that the two-photon wave function of entangled photons can be shaped by spectralphase manipulations exactly like classical pulses. The physical reason for this lies in the coherent spectrum of the two-photon state, which governs the temporal correlations between the photons in the very same way that the spectrum of a coherent pulse determines its shape.

Let us consider the two-photon state $|\Psi\rangle$ created by type-I, broadband down-conversion of a narrowband pump [9]:

$$
|\Psi\rangle \propto M|0\rangle+\int d \omega g(\omega)|\omega\rangle_{s}\left|\omega_{p}-\omega\right\rangle_{i}
$$

where $M \sim 1$, and the subscripts $s, i, p$ denote the signal, idler and pump modes, respectively. $g(\omega)$ is determined by the nonlinear coupling and the phase-matching conditions in the down-converting crystal, and exhibits a constant spectral phase for the case of perfect phase matching. At low photon fluxes, the two-photon wavefunction $\psi\left(t_{s}, t_{i}\right)$ represents the probability amplitude for the joint detection of signal and idler photons at times $t_{s}, t_{i}$ respectively, and is defined by:

$$
\psi\left(t_{s}, t_{i}\right)=\left\langle 0\left|E_{s}^{+} E_{i}^{+}\right| \Psi\right\rangle
$$

Introducing spectral filters $\Phi_{s}(\omega), \Phi_{i}(\omega)$ to the signal and idler modes, and assuming a gaussian spectrum with a bandwidth $\delta_{p}$ for the pump, the two-photon wavefunction can be approximated by [10, 11]:

$$
\psi\left(t_{s}, t_{i}\right) \propto e^{-\frac{1}{32} \delta_{p}^{2}\left(t_{s}+t_{i}\right)^{2}} G\left(t_{s}-t_{i}\right)
$$

where $G(t)$ is the inverse Fourier transform of $g(\omega) \Phi_{s}(\omega) \Phi_{i}\left(\omega_{p}-\omega\right)$ as a function of $\omega$. In other words, the two-photon wavefunction behaves like a coherent pulse with a spectrum $g(\omega)$, that was shaped by a spectral filter $\Phi(\omega)=\Phi_{s}(\omega) \Phi_{i}\left(\omega_{p}-\omega\right)$. This shaping can be nonlocal, since both the filtering and the detection of the signal and the idler photons can be located at different places. Note that similarly, the two-photon wavefunction of momentum entangled photons (see [12]) could be spatially shaped by phase manipulations in the momentum space. In collinear down-conversion (as in our case), it is convenient to define the higher (lower) energy photon as the signal (idler), or vice versa. In such a case $g(\omega)$ is symmetric about $\omega_{p} / 2$.

Although our discussion so far dealt with continuous down-conversion, shaping of the two-photon wavefunction is possible with pulsed down-conversion as well. In this case, the signal and the idler are both pulses with a constant (though undefined) spectral phase, like the pump. Thus, obviously, each can be shaped independently by spectral-phase filters, leading to the same result as in the continuous case [13].

The most direct way to observe the simultaneous arrival of two photons is to detect a nonlinear photonphoton interaction between them. Due to the extremely low efficiencies of nonlinear interactions with entangled photons, the detection of their inherent nonclassical correlations has been limited so far to photoelectric coincidences. Nonetheless, as was recently pointed-out [14], it is possible to generate surprisingly high fluxes of entangled photons without losing their nonclassical properties. Since the photons of each photon-pair are temporally correlated to within a time scale that is inversely proportional to their bandwidth $\Delta_{\mathrm{DC}}$, the maximal flux $\Phi_{\max }$ at which down-converted light can still be considered as composed of separated photon-pairs is:

$$
\Phi_{\text {max }} \approx \Delta_{\mathrm{DC}} .
$$

This flux corresponds to a mean spectral photondensity of $n=1$. For broadband down-conversion, $\Phi_{\max }$ can exceed $10^{13}$ photons per second, which corresponds to classically-high power levels of about $2 \mu W$. Such 


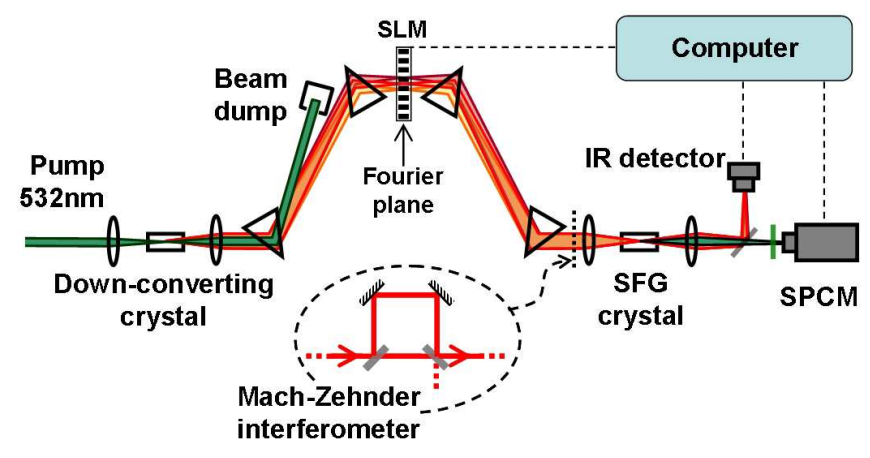

FIG. 1: Experimental layout. Entangled photons downconverted in one crystal are up-converted in the other crystal to produce the SFG photons. The symmetric imaging arrangement was designed to have a focus in the mid-way between the second and third prisms, creating there a spectral Fourier-plane. A computer-controlled SLM located at this plane was used to perform spectral-phase manipulations on the entangled photons. The SFG photons were counted using a single-photon counting module (SPCM-AQR-15 of $E G \& G)$, with a dark-count of $\sim 50 \mathrm{~s}^{-1}$. This dark-count was subtracted from all our measurements, which were performed with integration times of $5 s-10 s$. The entangledphotons beam was filtered-out from the SFG photons by a harmonic-separator mirror and filters, and its power was measured by a sensitive InGaAs detector. In order to demonstrate two-photon interference oscillations, a Mach-Zehnder interferometer was placed between the last prism and the SFG crystal.

ultrahigh fluxes of broadband entangled photons enable the demonstration of nonlinear interactions even with interaction efficiencies of the order of $10^{-9}$, which can be achieved without special enhancement mechanisms.

In this work we introduce the use of sum-frequency generation (SFG) as an ultrafast coincidence detector for photon fluxes that are below $\Phi_{\max }$. The sensitivity of the SFG process to relative delay between the photons is inversely proportional to the interaction's bandwidth, which for broadband phase-matching can be of the order of $\sim 100 \mathrm{fs}$. Such response times are completely unattainable with current electronic technologies, and allow a direct observation of the two-photon wavefunction.

Our experimental setup followed that of 14] and is depicted in Fig. 11 Basically, entangled photons generated by down-conversion of a continuous pump-laser $\left(\delta_{p} \approx 5 \mathrm{MHz}\right.$ around $\left.532 \mathrm{~nm}\right)$ in one crystal were imaged through a set of four Brewster-angle dispersion prisms onto a second crystal, to generate the SFG photons. This arrangement enabled a complete filtering-out of the pump with very low loss of down-converted photons; it also enabled a tuned compensation of dispersion (mainly of the crystals), thereby avoiding a significant reduction of the bandwidth that was effective for the SFG process. Finally, the spectral separation induced by the prisms made it possible for us to turn the entire optical lay-
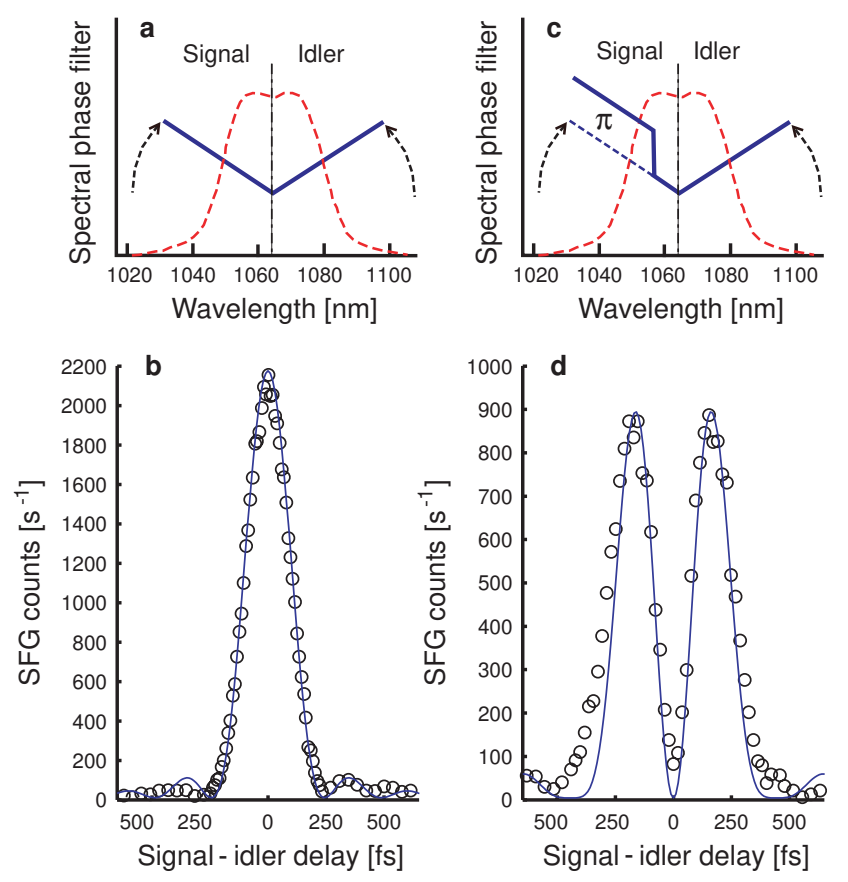

FIG. 2: Direct observation and shaping of the two-photon wavefunction. The relative delay between the signal and idler photons was induces by varying the slopes of the spectral phase filter described in (a). (b), The SFG counts (circles) and the absolute-square of the calculated two-photon wavefunction (line) as a function of the signal-idler delay. Shaping of the two-photon wavefunction was performed by adding a phase step-function in the middle of the signal spectrum, as described in (c). The SFG measurements of the shaped wavefunction are represented in $(\mathrm{d})$

out between the crystals into a pulse-shaper. Specifically, by using the lens after the first crystal to focus the beam at the mid-way between the second and third prisms, we created there a spectral Fourier plane. Then, by placing a computer-controlled spatial light-modulator (SLM) in that plane, we were able to introduce arbitrary phase shifts to different spectral-components of the entangled photons 15. The nonlinear crystals were periodically-poled $\mathrm{KTiOPO}_{4}$ (PPKTP) crystals, which were temperature-controlled to obtain broadband phase matching of $\Delta_{\mathrm{DC}} \approx 31 \mathrm{~nm}$ around $1064 \mathrm{~nm}$. This bandwidth implies $\Phi_{\max }=8.2 \cdot 10^{12} \mathrm{~s}^{-1}$, which corresponds to the classically-high power level of about $1.5 \mu \mathrm{W}$. Our measurements were performed at power levels of about $0.25 \mu W(n \approx 0.16)$.

To observe the two-photon wavefunction we introduced a relative delay between the signal and idler photons of each pair, by using the SLM to apply linear spectralphase functions with opposite slopes to the wavelengths below the degeneracy point of $1064 \mathrm{~nm}$ (signal) and above it (idler), as depicted in Fig. 2h. As described earlier, in this case of continuous, broadband down-conversion, a delay has no separate effect on neither the signal nor 
the idler photons. Nevertheless, as depicted in Fig. 20, the response of the SFG rate to the relative delay between the signal and the idler photons directly reflects the two-photon wavefunction. In order to demonstrate our ability to coherently shape the two-photon wavefunction we applied a phase step-function with amplitude of $\pi$ at the middle of the signal spectrum (roughly at $1057 \mathrm{~nm}$, see Fig 2r). With coherent pulses, this has the effect of splitting a pulse in time to two lobes. Figure $2 \mathrm{~d}$ depicts exactly such a behavior of the two-photon wavefunction. We note that these two lobes have opposite signs, which means there is a phase-shift between the events where the signal photon arrives before the idler and the events where it arrives after it. This phase shift, although undetectable by any measurement performed on the signal (or idler) photon itself, can be detected by interfering the SFG field with the pump laser.

It is important to understand under which conditions SFG can be considered a good measure for photon coincidences. The phase matching conditions in the nonlinear crystal dictate two relevant bandwidths (or three, in the non-degenerate case) for the process; one is the phasematched bandwidth of the input (low frequency) fields $\Delta_{\mathrm{LF}}$, and the other is the phase-matched bandwidth for the up-converted field $\delta_{\mathrm{UC}}$. Loosely speaking, for a SFG event to occur, three conditions must be satisfied. First, the spectrum of the input photons must lie within $\Delta_{\mathrm{LF}}$. Second, the spectrum of the up-converted photon must lie within $\delta_{\mathrm{Uc}}$. Third, the incoming photons must overlap temporally to within $\Delta_{\mathrm{LF}}^{-1}$. Obviously, only the third condition is equivalent to a coincidence detection. Therefore, SFG can be considered a coincidence detector only if the entire bandwidth $\Delta$ of the photons can be up-converted to a phase-matched wavelength, i.e. only if:

$$
\Delta_{\mathrm{LF}} \geq \Delta, \quad \delta_{\mathrm{UC}}>2 \Delta .
$$

Typically, the second condition in Eq. [5 does not hold, since in most cases $\delta_{\mathrm{UC}} \ll \Delta_{\mathrm{LF}}, \Delta$, especially when long crystals are utilized to increase the interaction efficiency. However, this condition can be circumvented with energy entangled photons. At low down-converted photon fluxes most of the SFG events are the result of up-conversion of entangled pairs. Specifically, the rate of SFG events with spontaneously down-converted light can be approximated by:

$$
R \propto \delta_{\mathrm{UC}} n^{2}+\Delta_{\mathrm{DC}} n^{2}+\Delta_{\mathrm{DC}} n .
$$

As described in [14], the last term, which exhibits a nonclassical linear intensity dependence and therefore dominates at $n \ll 1$, is the one that results from SFG of entangled pairs 16. Since the sum-energy of such pairs is always equal to the energy of the pump photons, for entangled-photons fluxes below $\Phi_{\max }$ the second condition of Eq. [5 can practically be replaced by:

$$
\delta_{\mathrm{UC}}>\delta_{p} .
$$

This condition is easily met for continuous downconversion, where the pump is typically very narrowband, as was in our case, where $\Delta_{\mathrm{LF}}=\Delta=\Delta_{\mathrm{DC}}$ (since identical crystals were used for the down- and upconversions), and $\delta_{\mathrm{UC}} \approx 0.1 \mathrm{~nm} \gg \delta_{p}$.

As is evident from Eq. [6] at high powers the contribution of entangled pairs to the overall SFG rate becomes negligible. All previous experiments that involved SFG with down-converted light [17, 18] were performed at extremely high powers $\left(\Phi \gg \Phi_{\max }\right)$. The observation and the temporal shaping of the coherent SFG process (denoted by the second term in Eq. 6) in these experiments are a direct result of the fact that $\delta_{\mathrm{UC}}$ was orders of magnitude smaller than the bandwidth of the light, thus violating the condition of Eq. 5 In such a case, many spectral combinations of the low-frequency fields contribute to the same narrowband up-converted field, and the resulting quantum interference can be controlled by manipulating the spectral phase of the incoming light, be it high-power down-converted light or shaped pulses. The effects observed in [17, 18] do not reflect coincidences (nor the fourth-order coherence of the light), and were demonstrated with shaped classical pulses as well [19]; had these experiments been performed with extremely ultrafast detectors (or with SFG with a large $\delta_{\mathrm{UC}}$ ), the coincidence rate would have registered mostly the regular bunching expected from thermally-distributed light.

The complementary aspect of the time and energy entanglement discussed earlier, is the fact that the coherence-time of the photon pairs $\left(\delta_{p}^{-1}\right)$ is much larger than the individual photons' coherence-time $\left(\Delta_{\mathrm{DC}}^{-1}\right)$, allowing two-photon interference even when the delay between the possible paths is too large to enable one-photon interference 1, 2, 20, 21, 22, 23. . To observe such an interference we placed a Mach-Zehnder interferometer in the entangled-photons path after the last prism (see Fig. 11). The interferometer was constructed by rotating the polarization of the entangled photons to $45^{\circ}$, utilizing the SLM to induce retardation between the vertical and horizontal polarizations, and then rotating the polarization $45^{\circ}$ back. In this configuration, the horizontal and vertical output polarizations are essentially the two output ports of the interferometer. In order to introduce retardations which were beyond the range of our SLM, we placed a $1 \mathrm{~mm}$ birefringent Calcite crystal immediately after the SLM, thus adding a constant retardation of about $163 \mu \mathrm{m}$.

The dependence of the coincidence rate on the delay $\tau$ between the interferometer arms is proportional to the absolute-square of the two-photon wavefunction, and can be represented by:

$$
R(\tau) \propto\left|\int g(\omega)\left(\cos \left(\omega_{o} \tau\right)+\cos \left(\left(\omega-\omega_{o}\right) \tau\right)\right) d \omega\right|^{2}
$$

where the symmetry of $g(\omega)$ about $\omega_{o}=\omega_{p} / 2$ was taken into account. Considering Eq. 8] we see that 


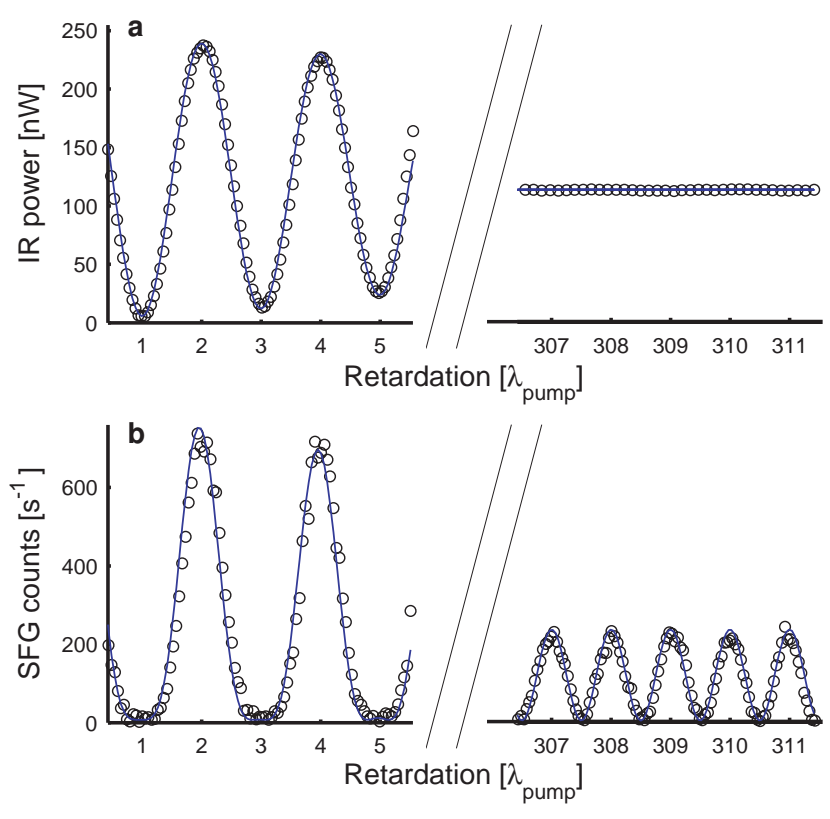

FIG. 3: Two-photon interference of time and energy entangled photons in a Mach-Zehnder interferometer (see Fig. 1). Interference oscillations of the IR power (a) and the SFG rate (b) as a function of the relative retardation between the interferometer arms. The experimental SFG counts (circles) are compared to the interference oscillations of the two-photon wavefunction, calculated according to Eq. 8 (line).

at small retardations the SFG rate is proportional to $\left|\left(\cos \left(\omega_{o} \tau\right)+1\right)\right|^{2}$, i.e. it is quadratically proportional to the IR intensity oscillations, as classically expected. For retardations which are beyond the single-photon coherence-length, the IR oscillations are washed out, and so is the second term in the integrand of Eq. 8] for the same reasons. Thus, the SFG rate becomes proportional to $\left|\cos \left(\omega_{o} \tau\right)\right|^{2}$, which oscillates with periodicity of $2 \omega_{o}=\omega_{p}$. The two-photon interference oscillations maintain their high visibility due to the long coherence length of the entangled pairs, which enables interference between the events at which a photon pair travelled unseparated along one interferometer arm, or the other. However, whenever such oscillations were detected with photoelectric coincidences 21, 23], the visibility dropped below $50 \%$ when the relative delay ranged between the coherence time of the photons $(\sim 100 \mathrm{fs})$ to the 4 orders of magnitude longer temporal resolution of the coincidence circuits $(\sim 1 n s)$. In contrast, the temporal resolution of the SFG process is equal to the coherence time of the photons since $\Delta_{\mathrm{LF}}=\Delta_{\mathrm{DC}}$. Thus, it performs as a 'background-free' coincidence detector, inherently rejecting non-coinciding photons. As can be seen in Fig. 3] the SFG counts follow the interference oscillations of the two-photon wavefunction throughout the entire range of delays, demonstrating visibility of $94 \% \pm 4 \%$ at a relative delay of $\sim 550 \mathrm{fs}$, where the single-photon interference completely dies-out. Note that $50 \%$ is the classical limit in the absence of first-order interference [21, 23, 24].

To conclude, we demonstrated how pulse-shaping techniques and SFG can be exploited to precisely control and measure the temporal properties of entangled photonpairs. Although we emphasized the ability of SFG to directly reflect the shape of the two-photon wavefunction, spectral-phase manipulations can be observed and utilized in schemes based on coincidence-measurements as well 25]. We believe both tools have potential applications in quantum measurement and quantum information science.

[1] Z. Y. Ou, et al. Phys. Rev. Lett. 65, 321 (1990).

[2] A. K. Ekert, et al. Phys. Rev. Lett. 69, 1293 (1992).

[3] K. Mattle, et al. Phys. Rev. Lett. 76, 4656 (1996).

[4] D. Bouwmeester, et al., Nature 390, 575 (1997).

[5] T. Jennewein, et al., Phys. Rev. Lett. 84, 4729 (2000).

[6] Z. Y. Ou and Y. J. Lu, Phys. Rev. Lett. 83, 2556 (1999).

[7] M. Bellini, et al., Phys. Rev. Lett. 90, 043602 (2003).

[8] S. Viciani, A. Zavatta, and M. Bellini, Phys. Rev. A. 69, 053801 (2004).

[9] Assuming low conversion efficiency, and neglecting the dependence of the down-converted spectrum on $\omega_{p}$.

[10] T. E. Keller and M. H. Rubin, Phys. Rev. A. 56, 1534 (1997).

[11] The bandwidth $\delta_{p}$ was taken as full-width at $1 / e$. Note that this analysis does not hold for type-II downconversion, where the wavefunction is broadened due to the group-velocity difference between the photons.

[12] C. H. Monken, P. H. Souto Ribeiro, and S. Pádua, Phys. Rev. A. 57, 3123 (1998).

[13] This description assumes the extreme case, where the pump is almost as broadband as the down-converted pulses.

[14] B. Dayan, A. Pe'er, A. A. Friesem, and Y. Silberberg, quant-ph/ 0411023 (2004), Accepted to Phys. Rev. Lett.

[15] A. M. Weiner, et al. Opt. Lett. 15, 326 (1990).

[16] Nonclassicality is exhibited by nondegenerate entangled photons at $n \ll 1$ since $\left|\mathrm{g}_{12}^{(2)}(0)\right|^{2}>\mathrm{g}_{11}^{(2)}(0) \mathrm{g}_{22}^{(2)}(0)$, violating the Cauchy-Schwartz inequality for classical fields.

[17] I. Abram, et al. Phys. Rev. Lett. 57, 2516 (1986).

[18] B. Dayan, et al. quant-ph/ 0302038 (2003).

[19] Z. Zheng and A. M. Weiner, Opt. Lett. 25, 984 (2000).

[20] J. D. Franson, Phys. Rev. A. 44, 4552 (1991).

[21] P. G. Kwiat, et al., Phys. Rev. A. 41, R2910 (1990).

[22] J. G. Rarity, et al., Phys. Rev. Lett. 65, 1348 (1990).

[23] J. Brendel, E. Mohler, and W. Martienssen, Phys. Rev. Lett. 66, 1142 (1991).

[24] C. Su and K. Wódkiewicz, Phys. Rev. A. 44, 6097 (1991).

[25] C. K. Hong, Z. Y. Ou, , and L. Mandel, Phys. Rev. Lett. 59, 2044 (1987). 